\title{
Effects of Early Transplantation of the Faecal Microbiota from Tibetan Pigs on the Gut Development of DSS-Challenged Piglets
}

\author{
H. Diao, ${ }^{1,2}$ Y. Xiao, ${ }^{1}$ H. L. Yan, ${ }^{1}$ B. Yu, ${ }^{1}$ J. He ${ }^{D},{ }^{1}$ P. Zheng, ${ }^{1}$ J. Yu, ${ }^{1}$ X. B. Mao ${ }^{1}{ }^{1}$ \\ and D. W. Chen $\left.{ }^{1}\right)^{1}$ \\ ${ }^{1}$ Institute of Animal Nutrition, Sichuan Agricultural University, Key Laboratory for Animal Disease-Resistance Nutrition of China \\ Ministry of Education, No. 46 Xinkang Road, Ya'an, Sichuan 625014, China \\ ${ }^{2}$ Animal Breeding and Genetics Key Laboratory of Sichuan Province, Sichuan Academy of Animal Science, No. 7 Niusha Road, \\ Chengdu, Sichuan 610066, China
}

Correspondence should be addressed to X. B. Mao; acatmxb2003@163.com and D. W. Chen; dwchen@sicau.edu.cn

H. Diao, Y. Xiao, and H. L. Yan contributed equally to this work.

Received 14 August 2020; Revised 30 December 2020; Accepted 6 January 2021; Published 19 January 2021

Academic Editor: Clara G. de los Reyes Gavilan

Copyright ( $2021 \mathrm{H}$. Diao et al. This is an open access article distributed under the Creative Commons Attribution License, which permits unrestricted use, distribution, and reproduction in any medium, provided the original work is properly cited.

\begin{abstract}
The present study was conducted to investigate the effects of early transplantation of the faecal microbiota from Tibetan pigs on the gut development of dextran sulphate sodium- (DSS-) challenged piglets. In total, 24 3-day-old DLY piglets were divided into four groups ( $n=6$ per group); a $2 \times 2$ factorial arrangement was used, which included faecal microbiota transplantation (FMT) (from Tibetan pigs) and DSS challenge. The whole trial lasted for 55 days. DSS infusion increased the intestinal density, serum diamine oxidase (DAO) activity, and colonic Escherichia coli count $(P<0.05)$, and decreased the Lactobacillus spp. count and mRNA abundances of epidermal growth factor (EGF), glucagon-like peptide-2 (GLP-2), insulin-like growth factor 1 (IGF-1), occludin, mucin 2 (MUC2), regeneration protein III $\gamma$ (RegIII $\gamma$ ), and interleukin-10 (IL-10) in the colon $(P<0.05)$. FMT increased the Lactobacillus spp. count and mRNA abundances of GLP-2, RegIII $\gamma$, and IL-10 in the colon $(P<0.05)$, and decreased the intestinal density, serum DAO activity, and colonic E. coli number $(P<0.05)$. In addition, in DSS-challenged piglets, FMT decreased the disease activity index $(P<0.05)$ and attenuated the effect of DSS challenge on the intestinal density, serum DAO activity, and colonic E. coli number $(P<0.05)$. These data indicated that the faecal microbiota from Tibetan pigs could attenuate the negative effect of DSS challenge on the gut development of piglets.
\end{abstract}

\section{Introduction}

Over thousands of years of evolution, hosts and bacteria have developed beneficial relationships, creating a mutually beneficial symbiotic environment [1]. The microbiota contribute to many physiological processes in hosts. In turn, hosts provide a basic developmental environment for microorganisms $[2,3]$. Recently, many studies in the biomedical field have shown that the intestinal microbiota are closely related to host health; these microorganisms can affect the digestion, absorption, and metabolism of nutrients, and regulate the physiological functions and the occurrence and development of diseases in hosts $[4,5]$.
The overall balance of gut microbial communities is important to ensure homeostasis in the intestinal mucosa $[6,7]$. The comparison between traditional and sterile animals has revealed the vital function of the intestinal microbiota in the development of the gastrointestinal tract profile, such as villus thickness, Peyer's patch maturity, and the numbers of isolated lymphoid follicles [8-11]. The gut microbiota can be used as targets for regulating metabolism and treating intestinal diseases in hosts [12]. Transplanting faecal materials from healthy individuals into patients with different diseases, such as inflammatory bowel disease, Clostridium difficile infection, metabolic diseases, and immune disorders, can be beneficial for the treatment of these diseases [13-16]. 
In animal production, the study of the relationship between the microbiota and host is still in its infancy.

We previously found huge differences in the gut microbiota composition among pig breeds [17]. Moreover, we found that transplantation of the faecal microbiota from Rongchang and Yorkshire pigs disrupted the normal microbial balance in the intestinal tract of suckling piglets, which was not conducive to the normal development of the intestinal tract [18]. In contrast, transplantation of the faecal microbiota from Tibetan pigs reduced diarrhoea and promoted absorption enzyme activities in piglets [18]. However, the potential anti-inflammatory value and application of the faecal microbiota from Tibetan pigs have been scarcely studied. Hence, the present study was conducted to investigate the protective effects of faecal microbiota transplantation (FMT) (from Tibetan pigs) on dextran sulphate sodium(DSS-) challenged suckling piglets in order to provide some new insights into the role of FMT in colitis.

\section{Materials and Methods}

2.1. Animals, Management, and Diets. Five Tibetan pigs (aged 12 weeks) were used in the present study as faecal donors. All the pigs were provided by a reservation farm and separately housed in individual metabolic cages in an environmentally controlled room for 8 weeks until sacrifice. The pigs were allowed ad libitum access to water and food. All the pigs received no antibiotics or probiotics during the latest 8-week period according to the standard for donor identification [19].

In total, 24 DLY suckling piglets $(2.08 \pm 0.12 \mathrm{~kg})$ were separated from their mothers at $48 \mathrm{~h}$ after birth, and were then fed with artificial milk for $24 \mathrm{~h}$. As a $2 \times 2$ factorial arrangement, all the piglets were randomly allotted to groups ( $n=6$ per group) matched for body weight and gender. The factors were FMT (piglets who received FMT from Tibetan pigs and those who did not) and challenge status (DSS-challenged and nonchallenged piglets). The four treatment groups were as follows: (1) the control (CTL) group, receiving only sterile PBS; (2) the CTL-T group, receiving FMT from Tibetan pigs; (3) the CTL-D group, receiving sterile PBS and DSS; and (4) the CTL-T-D group, receiving FMT from Tibetan pigs and DSS. All the piglets were artificially fed with milk substitutes in the first 15 days and then given solid feed in a gradual manner. The piglets in each group were separately housed in four environmentally controlled rooms and given the same diet throughout the process. The dietary compositions for each period are shown in Tables S1-S3. The entire trial lasted for 55 days.

\subsection{Faecal Microbiota Transplantation. Fresh faecal samples} were collected from five Tibetan pigs after $12 \mathrm{~h}$ of fasting and thoroughly mixed. Stool suspensions were prepared using a previously described method $[17,20]$. In brief, $1: 9(w / v)$ sterile saline was added to the mixed fresh faeces, following which the suspension was mixed and passed through stainless steel laboratory sieves $(2.0,1.0$, and $0.5 \mathrm{~mm}$, respectively). The piglets in the CTL-T and CTL-T-D groups were intragastrically infused with $10 \mathrm{~mL}$ faecal suspension daily for days 1-3 and every 2 days for days 4-15 and with $20 \mathrm{~mL}$ faecal suspension every 5 days for days 16-46.

2.3. DSS Administration. Experimental colitis was induced by the intragastric administration of DSS (MW: 36000-50000, MP Biomedicals, USA) according to a previously described method [21]. In brief, following $12 \mathrm{~h}$ fasting, the piglets in the CTL-D and CTL-T-D groups were intragastrically infused with $200 \mathrm{~mL}$ DSS solution (4\%) on day 51 and then with $100 \mathrm{~mL}$ DSS solution (4\%) daily for days 52-55. The nonchallenged piglets (CTL and CTL-T groups) were infused with an equal volume of sterile saline.

2.4. Sample Collection. On day 56, following $12 \mathrm{~h}$ fasting, $10 \mathrm{~mL}$ blood samples were collected from the precaval vein of each piglet, and the serum was isolated by centrifugation at 3,000 r/min for $10 \mathrm{~min}$. Following this, all the piglets were sacrificed by the injection of Zoletil 50 (BLESS Biotech, Beijing) at a dose of $10 \mathrm{mg} / \mathrm{kg}$ body weight and jugular exsanguinations. The length and weight of the intestine were measured after opening the abdomen. Sections of the jejunum, ileum, and colon were obtained, fixed in $10 \%$ buffered neutral formalin and embedded in paraffin for histological examination. Samples of the jejunum, ileum, and colon were immediately stored at $-80^{\circ} \mathrm{C}$ for analysing the antioxidant capacity and mRNA expression levels of some genes. Digesta samples of the cecum and colon were immediately isolated and stored at $-80^{\circ} \mathrm{C}$ for analysing the microbiota composition and metabolites.

2.5. Disease Activity Index (DAI). DAI was assessed according to the diarrhoea score, faecal occult blood index, and body weight change rate on a daily basis after DSS challenge; it was calculated by modifying a mixed clinical score described previously [22]. Each day during the challenge, the body weight of each piglet was measured, the diarrhoea score for each piglet was visually assessed according to the scoring system described by Hart and Dobb in 1988 [23], and the faecal occult blood index for each piglet was measured using faecal occult blood test paper strips (by the Colloidal Gold Method, W.H.P.M. Biotech, Beijing).

2.6. Histology of Intestine. Next, $1 \mathrm{~cm}$ long samples of the jejunum, ileum, and colon were fixed in $10 \%$ formaldehyde solution, dehydrated, and embedded in paraffin wax. The preserved samples were prepared after cutting, installing, and staining with haematoxylin and eosin and/or periodic acid-Schiff and Alcian blue. In total, 10 well-orientated sections of villus-crypt units in the jejunum and ileum were randomly selected, and the villus height and crypt depth were measured by using a light microscope (Olympus, Tokyo, Japan) and a digital microscope camera (Olympus Optical Company, Guangzhou, China). In addition, the number of goblet cells in the ileum and colon was counted using a previously described method [24].

2.7. Lipopolysaccharide (LPS) and Diamine Oxidase (DAO) Concentrations in the Serum. A porcine-specific ELISA kit (R\&D System, Minneapolis, MN) and a microplate reader (BioTek Instruments, Winooski, VT) were used to quantify the serum LPS concentration, in accordance with the 
manufacturer's protocol. The serum DAO concentration was measured using a commercial kit produced by Nanjing Jiancheng Bioengineering Institute (Jiangsu, China), and quantified by using a UV-vis spectrophotometer (UV1100, Shanghai, China).

2.8. Antioxidant Capacity and Glucagon-Like Peptide-2 (GLP-2) Level in the Jejunum and Colon. Supernatants of the jejunal and colonic samples were obtained according to the previously described methods [25]. In brief, the jejunum and colon were homogenised with sterile saline $(m / v=1 / 9)$ and centrifuged at $500 \times \mathrm{g}$ for $15 \mathrm{~min}$ at $4^{\circ} \mathrm{C}$. The supernatant was used for assessing the total protein concentration, total antioxidant capacity (T-AOC), malondialdehyde (MDA) concentration and superoxide dismutase (SOD) activity using commercial kits produced by Nanjing Jiancheng Bioengineering Institute (Jiangsu, China) in accordance with the manufacturer's protocol. The GLP-2 concentrations in the jejunum and colon were assessed using Pig EnzymeLinked Immunosorbent Assay Kits (R\&D System, Minneapolis, MN), and quantified using a BioTek Synergy HT Microplate Reader (BioTek Instruments, Winooski, VT).

2.9. Total RNA Extraction, Reverse Transcription Reaction, and Real-Time Quantitative PCR. Expression levels of targeted genes, including those encoding insulin-like growth factor 1 (IGF-1), GLP-2, epidermal growth factor (EGF), insulin-like growth factor 1 receptor (IGF-1R), angiogenin 4 (ANG4), mucin 1 (MUC1), regeneration protein III $\gamma$ $(\operatorname{RegIII} \gamma)$, mucin 2 (MUC2), zonula occludens 1 (ZO-1), occludin, interleukin-10 (IL-10), and interleukin-1 $\beta$ (IL-1 $\beta$ ) in the jejunum, ileum, and colon, were analysed by realtime PCR using the CFX96 Real-Time PCR Detection System (Bio-Rad, Richmond, CA) according to a previously described method [26]. In brief, total RNA was isolated from the frozen jejunum, ileum, and colon using the TRIzol Reagent (Takara Bio Inc., Dalian, China) in accordance with the manufacturer's protocols. Following this, RNA samples were reverse transcribed into complementary DNA (cDNA) using the PrimeScript ${ }^{\mathrm{TM}}$ RT reagent kit (Takara Bio Inc., Dalian, China). Finally, a $10 \mu \mathrm{L}$ quantitative fluorescent PCR reaction volume was used, which consisted of $1 \mu \mathrm{L}$ cDNA, $0.5 \mu \mathrm{L}$ upstream and downstream primers, $5 \mu \mathrm{L}$ SYBR Premix Ex Taq ${ }^{\mathrm{TM}}$, and $3 \mu \mathrm{L}$ RNase-free $\mathrm{H}_{2} \mathrm{O}$. The PCR cycle conditions were as follows: $30 \mathrm{~s}$ at $95^{\circ} \mathrm{C}, 10 \mathrm{~s}$ at $95^{\circ} \mathrm{C}$, and $25 \mathrm{~s}$ at $60^{\circ} \mathrm{C}$ for a total of 40 cycles. The primers shown in Table S4 were commercially synthesised by Invitrogen (Shanghai, China). The expression level of each gene in the tissues was calculated using $\beta$-actin as the reference gene.

2.10. Microbial Population Determination. The caecal and colonic digesta samples were used to extract bacterial DNA using Stool DNA kits (Omega Bio-tek, Doraville, CA). To quantify the microbial population, the primers and fluorescent oligonucleotide probes (Table S5) for total bacteria, Bacillus spp., Lactobacillus spp., Escherichia coli, and Bifidobacterium spp. were obtained according to the previously described methods [27, 28]. Quantitative real-time PCR was performed using the CFX96 Real-Time PCR Detection System (Bio-

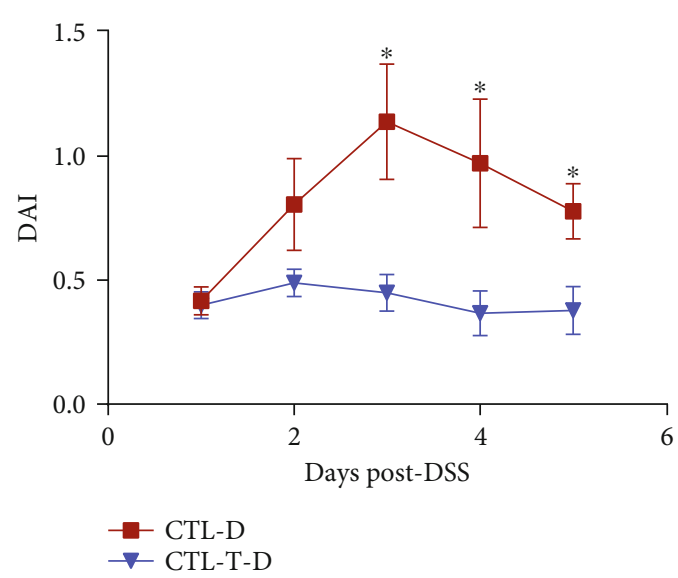

FIGURE 1: Effect of gut microbiota intervention on disease activity index (DAI) of dextran sulphate sodium- (DSS-) challenged piglets. CTL-D: piglets infused with sterile PBS and DSS. CTL-TD: piglets infused with the faecal microbiota from Tibetan pigs and DSS. ${ }^{*} P<0.05$.

Rad, CA, USA). A $25 \mu \mathrm{L}$ quantitative fluorescent PCR reaction volume was used for counting the total bacteria; it consisted of $1 \mu \mathrm{L}$ DNA, $1 \mu \mathrm{L}$ each of upstream and downstream primers, $12.5 \mu \mathrm{L}$ SYBR Premix Ex Taq ${ }^{\mathrm{TM}}$, and $9.5 \mu \mathrm{L} \mathrm{ddH}_{2} \mathrm{O}$. For counting the other bacteria, a $20 \mu \mathrm{L}$ PCR reaction volume was used; it consisted of $1 \mu \mathrm{L}$ DNA, $1 \mu \mathrm{L}$ each of upstream and downstream primers, $0.3 \mu \mathrm{L}$ probe, $1 \mu \mathrm{L}$ probe enhancer solution, $8 \mu \mathrm{L}$ RealMasterMix, and $7.7 \mu \mathrm{L} \mathrm{ddH}_{2} \mathrm{O}$. The PCR conditions and computing method were consistent with those reported by Qi et al. [27].

2.11. Short-Chain Fatty Acids (SCFAs). Frozen colonic digesta samples were used to measure SCFA levels using the Varian CP-3800 gas chromatographic system (Palo Alto, CA, USA), as described previously [29]. In brief, the colonic digesta were homogenised with distilled water $(m / v=1 / 1)$ and centrifuged at $500 \times \mathrm{g}$ for $10 \mathrm{~min}$. Then, $2 \mathrm{~mL}$ of supernatant was collected and centrifuged at $12,000 \times \mathrm{g}$ for $10 \mathrm{~min}$. Following this, $0.2 \mathrm{~mL}$ of $25 \%$ metaphosphoric acid was added to $1 \mathrm{~mL}$ of the supernatant, kept for $30 \mathrm{~min}$, and centrifuged at $12,000 \times \mathrm{g}$ for $10 \mathrm{~min}$. An equal volume of methanol was added to the supernatant and centrifuged at $12,000 \times \mathrm{g}$ for $10 \mathrm{~min}$. Finally, the supernatant was collected and stored at $-20^{\circ} \mathrm{C}$ for measuring the acetic acid, propionic acid, and butyric acid levels.

2.12. Statistical Analysis. The results are expressed as the means and SEM. Statistical analysis was performed by Student's $t$-test or two-way ANOVA using the statistical software SAS 8.2 (SAS Inst. Inc., NC); each piglet was the statistical unit. Significance was accepted at $P<0.05$, while $P<0.10$ was considered a tendency.

\section{Results}

3.1. DAI. On days 3-5, FMT (from Tibetan pigs) significantly decreased the DAI in the DSS-challenged piglets $(P<0.05$, Figure 1). 
TABLE 1: Effects of DSS challenge and FMT on the intestinal index in piglets.

\begin{tabular}{|c|c|c|c|c|c|c|c|c|}
\hline \multirow{2}{*}{ Items } & \multicolumn{2}{|c|}{ DSS- } & \multicolumn{2}{|c|}{ DSS +} & \multirow{2}{*}{ SEM } & \multicolumn{3}{|c|}{$P$ value } \\
\hline & Micro- & Micro+ & Micro- & Micro+ & & Micro & DSS & Micro $\times$ DSS \\
\hline Relative length of SI $(\mathrm{cm} / \mathrm{g})$ & 5.257 & 5.498 & 5.003 & 5.777 & 0.231 & 0.040 & 0.957 & 0.264 \\
\hline Relative length of LI $(\mathrm{cm} / \mathrm{g})$ & 1.195 & 1.208 & 1.092 & 1.217 & 0.056 & 0.234 & 0.409 & 0.333 \\
\hline Relative length of I (cm/g) & 6.453 & 6.073 & 6.095 & 6.993 & 0.273 & 0.049 & 0.902 & 0.250 \\
\hline Relative density of SI $(\mathrm{g} / \mathrm{cm})$ & 0.735 & 0.748 & 0.718 & 0.683 & 0.030 & 0.722 & 0.190 & 0.431 \\
\hline Relative density of LI $(\mathrm{g} / \mathrm{cm})$ & $1.673^{\mathrm{b}}$ & $1.688^{\mathrm{b}}$ & $2.125^{\mathrm{a}}$ & $1.707^{\mathrm{b}}$ & 0.083 & 0.024 & 0.010 & 0.016 \\
\hline Relative density of I (g/cm) & 0.908 & 0.918 & 0.972 & 0.858 & 0.035 & 0.156 & 0.963 & 0.094 \\
\hline Relative weight of SI (\%) & 3.862 & 4.058 & 3.598 & 3.958 & 0.186 & 0.150 & 0.341 & 0.666 \\
\hline Relative weight of LI (\%) & 2.003 & 2.005 & 2.315 & 2.060 & 0.115 & 0.284 & 0.126 & 0.277 \\
\hline Relative weight of I (\%) & 6.062 & 5.865 & 6.018 & 5.915 & 0.250 & 0.555 & 0.990 & 0.854 \\
\hline
\end{tabular}

TABLE 2: Effects of DSS challenge and FMT on the intestinal morphology and number of goblet cells in the intestines of piglets.

\begin{tabular}{|c|c|c|c|c|c|c|c|c|}
\hline \multirow{2}{*}{ Items } & \multicolumn{2}{|c|}{ DSS- } & \multicolumn{2}{|c|}{ DSS +} & \multirow{2}{*}{ SEM } & \multicolumn{3}{|c|}{$P$ value } \\
\hline & Micro- & Micro+ & Micro- & Micro+ & & Micro & DSS & Micro $\times$ DSS \\
\hline \multicolumn{9}{|l|}{ Jejunum } \\
\hline Villus height $(\mu \mathrm{m})$ & $521.360^{\mathrm{b}}$ & $546.800^{\mathrm{b}}$ & $1168.700^{\mathrm{a}}$ & $485.680^{\mathrm{b}}$ & 70.195 & $<0.001$ & $<0.001$ & $<0.001$ \\
\hline Crypt depth $(\mu \mathrm{m})$ & $229.620^{\mathrm{b}}$ & $210.290^{\mathrm{b}}$ & $554.230^{\mathrm{a}}$ & $218.530^{\mathrm{b}}$ & 35.267 & $<0.001$ & $<0.001$ & $<0.001$ \\
\hline Villus height: crypt depth & 2.306 & 2.631 & 2.112 & 2.254 & 0.077 & 0.115 & 0.059 & 0.524 \\
\hline \multicolumn{9}{|l|}{ Ileum } \\
\hline Villus height $(\mu \mathrm{m})$ & $609.830^{\mathrm{b}}$ & $563.870^{\mathrm{b}}$ & $1161.670^{\mathrm{a}}$ & $442.720^{\mathrm{b}}$ & 69.900 & $<0.001$ & 0.004 & $<0.001$ \\
\hline Crypt depth $(\mu \mathrm{m})$ & $244.020^{\mathrm{b}}$ & $229.960^{\mathrm{b}}$ & $528.700^{\mathrm{a}}$ & $181.300^{\mathrm{b}}$ & 33.556 & $<0.001$ & $<0.001$ & $<0.001$ \\
\hline Villus height: crypt depth & 2.615 & 2.464 & 2.195 & 2.497 & 0.084 & 0.655 & 0.259 & 0.190 \\
\hline \multicolumn{9}{|l|}{ Goblet cells } \\
\hline Ileum & 69.875 & 75.600 & 73.875 & 71.981 & 2.201 & 0.688 & 0.968 & 0.427 \\
\hline Colon & $102.197^{\mathrm{a}}$ & $100.656^{\mathrm{a}}$ & $83.200^{\mathrm{b}}$ & $74.200^{\mathrm{b}}$ & 3.348 & 0.237 & $<0.001$ & 0.397 \\
\hline
\end{tabular}

3.2. Intestinal Index. The effects of FMT and DSS challenge on the intestinal index of the piglets are shown in Table 1. DSS challenge increased the large intestinal density of the piglets $(P<0.05)$. In the piglets, FMT decreased the large intestinal density and increased the length of the small intestine and whole intestine $(P<0.05)$. Moreover, FMT attenuated the effect of DSS challenge on the density of the large intestine $(P<0.05)$ and whole intestine $(P=0.094)$.

DSS-: infused with sterile saline; DSS+: infused with DSS; Micro-: infused with sterile saline; Micro+: infused with the faecal microbiota from Tibetan pigs; SI: small intestine; LI: large intestine; I: whole intestine. ${ }^{\mathrm{a}-\mathrm{b}} \mathrm{W}$ ithin a row, means without a common superscript differ $(P<0.05)$.

3.3. Intestinal Morphology and Number of Goblet Cells. The villus height and crypt depth in the jejunum and ileum and the number of goblet cells in the ileum and colon are indicated in Table 2. In the piglets, DSS challenge increased the villus height and crypt depth in the jejunum and ileum and decreased the villus height:crypt depth ratio in the jejunum and the number of goblet cells in the colon $(P<0.05)$. FMT decreased the villus height and crypt depth in the jejunum and ileum of the piglets $(P<0.05)$. Moreover, FMT relieved the effect of DSS chal- lenge on the villus height and crypt depth in the jejunum and ileum of the piglets $(P<0.05)$.

DSS-: infused with sterile saline; DSS+: infused with DSS; Micro-: infused with sterile saline; Micro+: infused with the faecal microbiota from Tibetan pigs. ${ }^{a-b}$ Within a row, means without a common superscript differ $(P<0.05)$.

3.4. Relative mRNA Expression Levels of Intestinal Development-Related Genes and GLP-2 Concentrations. As shown in Table 3, DSS challenge decreased the mRNA expression of ANG4 in the jejunum $(P<0.05)$. It also decreased the mRNA expressions levels of EGF, GLP-2, ANG4, and IGF-1 in the colon $(P<0.05)$, and the GLP-2 concentrations in the jejunum $(P<0.05)$ and colon $(P=0.099)$ of the piglets. FMT increased the mRNA expression level of ANG4 in the jejunum $(P<0.05)$ and the mRNA expression levels and concentrations of GLP-2 in the jejunum and colon of the piglets $(P<0.05)$. However, no significant interaction effects were noted with regard to the mRNA expression levels of intestinal development-related genes and GLP-2 concentrations between DSS administration and FMT $(P>0.05)$.

DSS-: infused with sterile saline; DSS+: infused with DSS; Micro-: infused with sterile saline; Micro+: infused with the faecal microbiota from Tibetan pigs; EGF: epidermal growth 
TABLE 3: Effects of DSS challenge and FMT on the mRNA expression levels of intestinal development-related genes and GLP-2 concentrations in the intestines of piglets.

\begin{tabular}{|c|c|c|c|c|c|c|c|c|}
\hline \multirow{2}{*}{ Items } & \multicolumn{2}{|c|}{ DSS- } & \multicolumn{2}{|c|}{ DSS+ } & \multirow{2}{*}{ SEM } & \multicolumn{3}{|c|}{$P$ value } \\
\hline & Micro- & Micro+ & Micro- & Micro+ & & Micro & DSS & Micro $\times$ DSS \\
\hline \multicolumn{9}{|l|}{ Jejunum } \\
\hline EGF & 1.000 & 1.071 & 0.913 & 0.911 & 0.052 & 0.749 & 0.263 & 0.740 \\
\hline GLP-2 & 1.000 & 1.346 & 0.775 & 1.161 & 0.087 & 0.034 & 0.740 & 0.903 \\
\hline ANG4 & $1.000^{\mathrm{ab}}$ & $1.344^{\mathrm{a}}$ & $0.624^{\mathrm{b}}$ & $0.935^{\mathrm{ab}}$ & 0.074 & 0.008 & 0.002 & 0.887 \\
\hline IGF-1 & 1.000 & 1.145 & 0.887 & 1.022 & 0.067 & 0.323 & 0.402 & 0.972 \\
\hline IGF-1R & 1.000 & 1.085 & 0.979 & 0.984 & 0.036 & 0.553 & 0.426 & 0.601 \\
\hline \multicolumn{9}{|l|}{ Ileum } \\
\hline EGF & 1.000 & 1.190 & 0.911 & 0.979 & 0.047 & 0.192 & 0.129 & 0.465 \\
\hline GLP-2 & 1.000 & 1.178 & 1.161 & 1.000 & 0.037 & 0.107 & 0.107 & 0.390 \\
\hline ANG4 & 1.000 & 1.085 & 0.935 & 0.967 & 0.053 & 0.571 & 0.345 & 0.667 \\
\hline IGF-1 & 1.000 & 1.074 & 1.022 & 0.956 & 0.043 & 0.589 & 0.309 & 0.786 \\
\hline IGF-1R & 1.000 & 1.019 & 0.984 & 1.002 & 0.026 & 0.375 & 0.393 & 0.586 \\
\hline \multicolumn{9}{|l|}{ Colon } \\
\hline EGF & 1.000 & 1.352 & 0.515 & 0.787 & 0.129 & 0.207 & 0.040 & 0.870 \\
\hline GLP-2 & $1.000^{\mathrm{ab}}$ & $1.599^{\mathrm{a}}$ & $0.348^{\mathrm{b}}$ & $1.182^{\mathrm{ab}}$ & 0.136 & 0.003 & 0.020 & 0.585 \\
\hline ANG4 & 1.000 & 1.081 & 0.700 & 0.607 & 0.071 & 0.963 & 0.005 & 0.491 \\
\hline IGF-1 & $1.000^{\mathrm{ab}}$ & $1.215^{\mathrm{a}}$ & $0.656^{\mathrm{b}}$ & $0.701^{\mathrm{b}}$ & 0.068 & 0.228 & 0.001 & 0.427 \\
\hline IGF-1R & 1.000 & 1.137 & 0.902 & 0.993 & 0.081 & 0.509 & 0.485 & 0.896 \\
\hline \multicolumn{9}{|c|}{ GLP-2 concentration (pmol/gprot) } \\
\hline Jejunum & $3.052^{\mathrm{ab}}$ & $3.260^{\mathrm{a}}$ & $2.856^{\mathrm{b}}$ & $2.936^{\mathrm{b}}$ & 0.046 & 0.061 & 0.002 & 0.386 \\
\hline Colon & $2.721^{\mathrm{ab}}$ & $3.594^{\mathrm{a}}$ & $2.164^{\mathrm{b}}$ & $3.288^{\mathrm{ab}}$ & 0.269 & 0.002 & 0.099 & 0.724 \\
\hline
\end{tabular}

TABLE 4: Effects of DSS challenge and FMT on the colonic antioxidant capacity of piglets.

\begin{tabular}{|c|c|c|c|c|c|c|c|c|}
\hline \multirow{2}{*}{ Items } & \multicolumn{2}{|c|}{ DSS- } & \multicolumn{2}{|c|}{ DSS +} & \multirow{2}{*}{ SEM } & \multicolumn{3}{|c|}{$P$ value } \\
\hline & Micro- & Micro+ & Micro- & Micro+ & & Micro & DSS & Micro $\times$ DSS \\
\hline \multicolumn{9}{|l|}{ Jejunum } \\
\hline MDA (nmol/mg protein) & 0.863 & 0.930 & 1.127 & 1.029 & 0.038 & 0.818 & 0.015 & 0.242 \\
\hline T-AOC (U/mg protein) & 0.259 & 0.296 & 0.214 & 0.288 & 0.013 & 0.029 & 0.266 & 0.446 \\
\hline SOD (U/mg protein) & 88.654 & 98.499 & 77.264 & 92.747 & 16.146 & 0.053 & 0.179 & 0.652 \\
\hline \multicolumn{9}{|l|}{ Colon } \\
\hline MDA (nmol/mg protein) & $1.339^{\mathrm{a}}$ & $1.016^{\mathrm{b}}$ & $1.583^{\mathrm{a}}$ & $1.025^{\mathrm{b}}$ & 0.056 & $<0.001$ & 0.014 & 0.021 \\
\hline T-AOC (U/mg protein) & $0.328^{\mathrm{ab}}$ & $0.422^{\mathrm{a}}$ & $0.242^{\mathrm{b}}$ & $0.387^{\mathrm{a}}$ & 0.061 & 0.554 & 0.077 & 0.126 \\
\hline SOD (U/mg protein) & 133.097 & 126.493 & 110.154 & 124.733 & 6.044 & 0.745 & 0.032 & 0.016 \\
\hline
\end{tabular}

factor; IGF-1: insulin-like growth factor 1; GLP-2: glucagonlike peptide 2; IGF-1R: insulin-like growth factor 1 receptor; ANG4: angiogenin $4 .{ }^{\mathrm{a}-\mathrm{b}} \mathrm{Within}$ a row, means without a common superscript differ $(P<0.05)$.

3.5. Intestinal Antioxidant Capacity. The antioxidant capacity in the jejunum and colon of the piglets is summarised in Table 4. DSS challenge increased the MDA concentrations in the jejunum and colon $(P<0.05)$ and decreased the TAOC capacity and SOD activity in the colon of the piglets $(P<0.05)$. FMT increased the T-AOC capacity and SOD activity in the jejunum $(P<0.05)$ and decreased the MDA concentration in the colon of the piglets $(P<0.05)$. In addition, FMT attenuated the effect of DSS challenge on the
MDA concentration and SOD activity in the colon of the piglets $(P<0.05)$.

DSS-: infused with sterile saline; DSS+: infused with DSS; Micro-: infused with sterile saline; Micro+: infused with the faecal microbiota from Tibetan pigs; T-AOC: total antioxidant capacity; MDA: malondialdehyde; SOD: superoxide dismutase. ${ }^{\mathrm{a}-\mathrm{b}}$ Within a row, means without a common superscript differ $(P<0.05)$.

3.6. Intestinal Barrier Function. As shown in Table 5, DSS challenge increased the serum DAO activity $(P<0.05)$ and enhanced the mRNA expression level of IL- $1 \beta$ in the colon $(P<0.05)$. Moreover, it decreased the mRNA expression levels of MUC1 and MUC2 in the jejunum $(P<0.05)$ and 
TABLE 5: Effects of DSS challenge and FMT on the serum DOA activity and LPS concentration and on the mRNA expression levels of intestinal barrier-related genes in the intestines of piglets.

\begin{tabular}{|c|c|c|c|c|c|c|c|c|}
\hline \multirow{3}{*}{ Jejunum } & \multicolumn{2}{|c|}{ DSS- } & \multicolumn{2}{|c|}{ DSS +} & \multirow{2}{*}{ SEM } & \multicolumn{3}{|c|}{$P$ value } \\
\hline & Micro- & Micro+ & Micro- & Micro+ & & Micro & DSS & Micro $\times$ DSS \\
\hline & & & & & & & & \\
\hline Occludin & 1.000 & 0.946 & 0.945 & 0.859 & 0.034 & 0.327 & 0.319 & 0.826 \\
\hline $\mathrm{ZO}-1$ & 1.000 & 1.024 & 0.992 & 0.933 & 0.035 & 0.819 & 0.508 & 0.580 \\
\hline MUC1 & $1.000^{\mathrm{ab}}$ & $1.186^{\mathrm{a}}$ & $0.316^{\mathrm{b}}$ & $0.364^{\mathrm{b}}$ & 0.088 & 0.159 & $<0.001$ & 0.397 \\
\hline MUC2 & 1.000 & 1.010 & 0.545 & 0.570 & 0.083 & 0.908 & 0.007 & 0.962 \\
\hline $\operatorname{RegIII} \gamma$ & 1.000 & 1.167 & 0.804 & 0.974 & 0.067 & 0.215 & 0.155 & 0.991 \\
\hline Ileum & & & & & & & & \\
\hline Occludin & 1.000 & 0.965 & 0.929 & 0.867 & 0.055 & 0.680 & 0.475 & 0.906 \\
\hline $\mathrm{ZO}-1$ & 1.000 & 1.011 & 0.910 & 0.962 & 0.042 & 0.728 & 0.441 & 0.819 \\
\hline MUC1 & 1.000 & 1.119 & 1.119 & 0.898 & 0.049 & 0.723 & 0.176 & 0.402 \\
\hline MUC2 & 1.000 & 0.980 & 0.962 & 1.049 & 0.059 & 0.794 & 0.903 & 0.677 \\
\hline $\operatorname{RegIII} \gamma$ & 1.000 & 1.023 & 0.940 & 0.919 & 0.053 & 0.992 & 0.468 & 0.845 \\
\hline Colon & & & & & & & & \\
\hline Occludin & $1.000^{\mathrm{a}}$ & $0.890^{\mathrm{a}}$ & $0.623^{\mathrm{b}}$ & $0.609^{\mathrm{b}}$ & 0.047 & 0.364 & $<0.001$ & 0.484 \\
\hline $\mathrm{ZO}-1$ & 1.000 & 0.915 & 0.747 & 0.766 & 0.064 & 0.803 & 0.137 & 0.690 \\
\hline MUC1 & 1.000 & 1.060 & 0.844 & 1.025 & 0.044 & 0.180 & 0.285 & 0.495 \\
\hline MUC2 & $1.000^{\mathrm{ab}}$ & $1.244^{\mathrm{a}}$ & $0.717^{\mathrm{b}}$ & $0.801^{\mathrm{b}}$ & 0.114 & 0.165 & 0.005 & 0.490 \\
\hline $\operatorname{RegIII} \gamma$ & $1.000^{\mathrm{a}}$ & $1.030^{\mathrm{a}}$ & $0.265^{\mathrm{c}}$ & $0.555^{\mathrm{b}}$ & 0.074 & 0.035 & $<0.001$ & 0.082 \\
\hline IL-1 $\beta$ & $1.000^{\mathrm{b}}$ & $0.839^{\mathrm{b}}$ & $1.631^{\mathrm{a}}$ & $1.182^{\mathrm{b}}$ & 0.074 & 0.003 & $<0.001$ & 0.120 \\
\hline IL-10 & $1.000^{\mathrm{c}}$ & $1.531^{\mathrm{ab}}$ & $1.228^{\mathrm{bc}}$ & $1.739^{\mathrm{a}}$ & 0.080 & 0.001 & 0.074 & 0.931 \\
\hline Serum & & & & & & & & \\
\hline $\mathrm{DAO}(\mathrm{U} / \mathrm{L})$ & 10.418 & 9.987 & 11.492 & 10.476 & 0.140 & $<0.001$ & $<0.001$ & 0.099 \\
\hline LPS (ng/mL) & 68.155 & 67.322 & 70.929 & 69.472 & 1.208 & 0.654 & 0.340 & 0.903 \\
\hline
\end{tabular}

the mRNA expression levels of occludin, MUC2, RegIII $\gamma$, and IL-10 in the colon of the piglets $(P<0.05)$. FMT decreased the serum DAO activity $(P<0.05)$, downregulated the mRNA expression level of IL- $1 \beta$ in the colon $(P<0.05)$, and upregulated the mRNA expression levels of RegIII $\gamma$ and IL-10 in the colon of the piglets $(P<0.05)$. FMT also attenuated the effect of DSS challenge on the serum DAO activity $(P<0.05)$ and the mRNA expression level of RegIII $\gamma$ in the colon $(P=0.082)$ of the piglets.

DSS-: infused with sterile saline; DSS+: infused with DSS; Micro-: infused with sterile saline; Micro+: infused with the faecal microbiota from Tibetan pigs; ZO-1: zonula occludens 1; MUC1: mucin 1; MUC2: mucin 2; REGIII $\gamma$ : regeneration protein III $\gamma$; IL-10: interleukin-10; IL-1 $\beta$ : interleukin-1 $\beta$; LPS: lipopolysaccharide; DAO: diamine oxidase. ${ }^{\mathrm{a}-\mathrm{b}} \mathrm{Within}$ a row, means without a common superscript differ $(P<0.05)$.

As shown in Tables 6 and 7, DSS challenge increased the E. coli count and the propionic acid and total SCFA levels $(P<0.05)$ and decreased the Lactobacillus spp. count and the butyric acid level $(P<0.05)$ in the colonic digesta of the piglets. FMT increased the Lactobacillus spp. and Bacillus spp. counts in the caecal digesta and the total bacteria and Lactobacillus spp. counts in the colonic digesta $(P<0.05)$. It decreased the $E$. coli counts in the caecal and colonic digesta and the acetic acid, propionic acid, and total SCFA levels in the colonic digesta of the piglets $(P<0.05)$. Moreover, FMT attenuated the effect of DSS challenge on the E. coli count $(P=0.072)$ and the acetic acid $(P<0.05)$, propionic acid $(P<0.05)$, and total SCFA $(P<0.05)$ levels in the colonic digesta of the piglets.

DSS-: infused with sterile saline; DSS+: infused with DSS; Micro-: infused with sterile saline; Micro+: infused with the faecal microbiota from Tibetan pigs. ${ }^{\mathrm{a}-\mathrm{b}}$ Within a row, means without a common superscript differ $(P<0.05)$.

DSS-: infused with sterile saline; DSS+: infused with DSS; Micro-: infused with sterile saline; Micro+: infused with the faecal microbiota from Tibetan pigs. ${ }^{\mathrm{a}-\mathrm{b}} \mathrm{W}$ ithin a row, means without a common superscript differ $(P<0.05)$.

\section{Discussion}

The number of intestinal microbes is dynamically balanced, and an imbalance can cause an inflammatory response by the immune system [30]. Colitis is a common gastrointestinal dysfunction disease, and it is clinically manifested as diarrhoea and bloody stools, which are associated with an abnormal immune response induced by intestinal microbiome disorders [31]. In the present study, in order to explore the potential benefits of FMT from Tibetan pigs in an inflammatory model and its application value in animal husbandry production, DSS was orally infused in piglets. DSS challenge increased the DAI, visible bloody stools, and diarrhoea in the 
TABLE 6: Effects of DSS challenge and FMT on the caecal and colonic E. coli, Lactobacillus spp., Bifidobacterium spp., Bacillus spp., and total bacterial counts in caecal and colonic digesta of piglets $(\log (\operatorname{copies} / g))$.

\begin{tabular}{|c|c|c|c|c|c|c|c|c|}
\hline \multirow{2}{*}{ Items } & \multicolumn{2}{|c|}{ DSS- } & \multicolumn{2}{|c|}{ DSS +} & \multirow{2}{*}{ SEM } & \multicolumn{3}{|c|}{$P$ value } \\
\hline & Micro- & Micro+ & Micro- & Micro+ & & Micro & DSS & Micro $\times$ DSS \\
\hline \multicolumn{9}{|l|}{ Cecum } \\
\hline Total bacteria & 11.503 & 11.422 & 11.481 & 11.422 & 0.028 & 0.150 & 0.665 & 0.958 \\
\hline Bacillus spp. & 9.777 & 9.898 & 9.798 & 9.943 & 0.026 & 0.011 & 0.493 & 0.805 \\
\hline Lactobacillus spp. & $8.345^{\mathrm{b}}$ & $8.965^{\mathrm{a}}$ & $8.145^{\mathrm{b}}$ & $8.707^{\mathrm{ab}}$ & 0.146 & 0.001 & 0.150 & 0.851 \\
\hline E. coli & $8.574^{\mathrm{ab}}$ & $8.157^{\mathrm{b}}$ & $9.078^{\mathrm{a}}$ & $8.088^{\mathrm{b}}$ & 0.147 & 0.001 & 0.252 & 0.134 \\
\hline Bifidobacterium spp. & 8.021 & 8.006 & 7.898 & 8.007 & 0.037 & 0.549 & 0.440 & 0.434 \\
\hline \multicolumn{9}{|l|}{ Colon } \\
\hline Total bacteria & $11.532^{\mathrm{ab}}$ & $11.670^{\mathrm{a}}$ & $11.423^{\mathrm{b}}$ & $11.736^{\mathrm{a}}$ & 0.037 & 0.001 & 0.706 & 0.137 \\
\hline Bacillus spp. & 9.933 & 10.066 & 9.836 & 9.939 & 0.037 & 0.113 & 0.131 & 0.833 \\
\hline Lactobacillus spp. & $8.475^{\mathrm{bc}}$ & $9.388^{\mathrm{a}}$ & $7.878^{\mathrm{c}}$ & $8.731^{\mathrm{ab}}$ & 0.181 & $<0.001$ & 0.003 & 0.875 \\
\hline E. coli & $8.740^{\mathrm{ab}}$ & $8.153^{\mathrm{b}}$ & $9.507^{\mathrm{a}}$ & $8.274^{\mathrm{b}}$ & 0.131 & $<0.001$ & 0.007 & 0.072 \\
\hline Bifidobacterium spp. & 7.629 & 7.931 & 7.798 & 7.801 & 0.052 & 0.142 & 0.848 & 0.152 \\
\hline
\end{tabular}

TABLE 7: Effects of DSS challenge and FMT on SCFAs levels in the colonic digesta of piglets $(\mu \mathrm{mol} / \mathrm{g})$.

\begin{tabular}{|c|c|c|c|c|c|c|c|c|}
\hline \multirow{2}{*}{ Items } & \multicolumn{2}{|c|}{ DSS- } & \multicolumn{2}{|c|}{ DSS +} & \multirow{2}{*}{ SEM } & \multicolumn{3}{|c|}{$P$ value } \\
\hline & Micro- & Micro+ & Micro- & Micro+ & & Micro & DSS & Micro $\times$ DSS \\
\hline Acetic acid & $55.828^{\mathrm{b}}$ & $53.410^{\mathrm{b}}$ & $63.297^{\mathrm{a}}$ & $51.716^{\mathrm{b}}$ & 1.214 & $<0.001$ & 0.103 & 0.014 \\
\hline Propionic acid & $31.384^{\mathrm{b}}$ & $29.340^{\mathrm{b}}$ & $42.086^{\mathrm{a}}$ & $27.223^{\mathrm{b}}$ & 1.310 & $<0.001$ & 0.001 & $<0.001$ \\
\hline Butyric acid & 16.973 & 16.544 & 15.132 & 15.524 & 0.335 & 0.978 & 0.036 & 0.527 \\
\hline Total volatile fatty acid & $104.185^{\mathrm{b}}$ & $99.294^{\mathrm{bc}}$ & $120.514^{\mathrm{a}}$ & $94.463^{\mathrm{c}}$ & 2.353 & $<0.001$ & 0.033 & $<0.001$ \\
\hline
\end{tabular}

piglets, particularly in those without FMT. In addition, DSS infusion impaired the gut histology, development, antioxidant capacity, and barrier function. These results were consistent with those of previous studies in mice, indicating that our colitis model was successful [32-34].

In the present study, we also found that FMT from Tibetan pigs significantly reduced the DAI in the DSS-challenged piglets, indicating that early FMT from Tibetan pigs can alleviate the clinical symptoms induced by DSS infusion. Lactobacillus acidophilus can reduce the increase in the DAI and colonic histopathology scores triggered by DSS challenge in mice and has a therapeutic effect on acute ulcerative colitis [35]. Similarly, colitis induced by DSS challenge can be relieved by Bifidobacterium treatment in the mice with a decreasing diarrhoea score and colonic hyperaemia [36]. In our previous study, 16S rRNA gene sequencing demonstrated that the gut microbiota profile differs among three pig breeds (Yorkshire pigs, Tibetan pigs, and Rongchang pigs), with higher Lactobacillus spp. and Parabacteroides spp. counts being observed in Tibetan pigs [17]. Lactobacillus and Parabacteroides are two genera considered to be positively correlated with the cure of colitis $[37,38]$. The present study also revealed that the piglets who received the stool suspensions from Tibetan pigs had higher copies of Lactobacillus spp. in their colonic digesta, which may be an important reason why DSS-induced colitis is relieved by FMT from Tibetan pigs.

As DSS primarily causes colon damage, most studies have focused on the large intestine, with few studies focusing on the small intestine. In the present study, DSS challenge increased the large intestinal density, villus height, and crypt depth in the jejunum and ileum and decreased the villus height:crypt depth ratio in the jejunum and the mRNA expression levels of EGF, GLP-2, ANG4, and IGF-1 in the colon of the piglets. Thus, DSS challenge not only impaired the development of the colon but also negatively affected the jejunum and ileum of the piglets. We also found that FMT attenuated the effect of DSS challenge on the large intestinal density, villus height, and crypt depth in the jejunum and the GLP-2 concentrations in the jejunum and colon of the piglets. It is well known that EGF and IGF-1 are the main regulators of intestinal cell proliferation [39, 40]. ANG4 is a Paneth cell granule protein that plays an important role in shaping intestinal angiogenesis [41]. GLP-2, a specific growth regulator of intestinal epithelial cells, is a hormone that is mainly synthesised and secreted by intestinal endocrine cells. It stimulates the intestinal blood flow and intestinal cell proliferation and thus promotes the growth of the intestinal mucosa and improves nutrient absorption [42-44]. Changes in the intestinal microbiota composition would affect endogenous GLP-2 production [45]. Therefore, early FMT from Tibetan pigs can attenuate the negative effect of DSS infusion on the intestinal development of piglets, which may be associated with the GLP-2 production.

The intestinal barrier function plays an important role in gut health; the effects of DSS challenge and FMT on gut health are mainly reflected in the intestinal barrier function. In the inner barrier of the intestinal mucosa, the tight junction is mainly composed of the peripheral membrane protein 


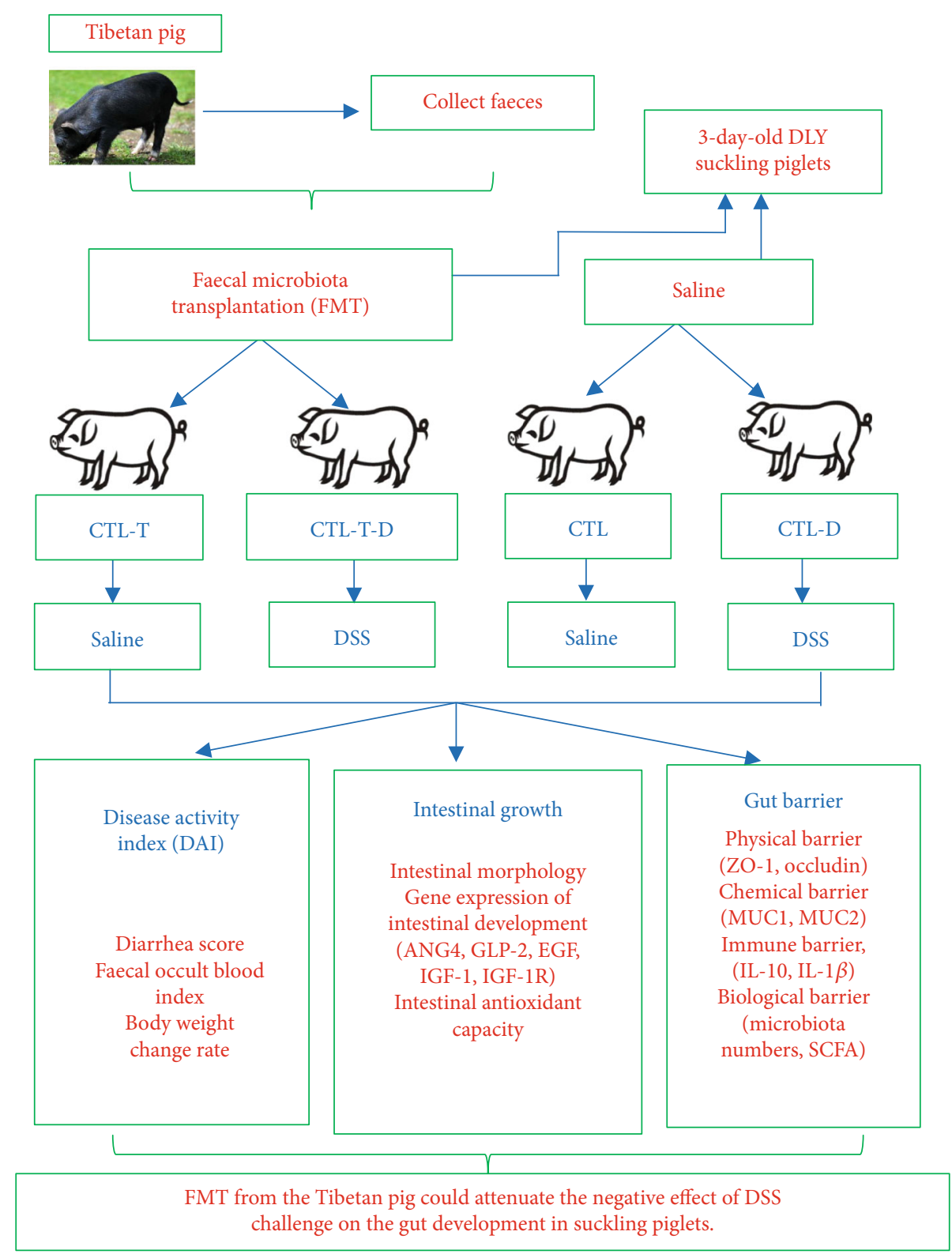

FIgURE 2: The overall frame diagram. CTL: receiving only sterile PBS. CTL-T: receiving the faecal microbiota from Tibetan pigs. CTL-D: receiving sterile PBS, followed by treatment with dextran sulphate sodium (DSS). CTL-T-D: receiving the faecal microbiota from Tibetan pigs, followed by treatment with DSS.

(ZO family) and transmembrane protein (occludin and claudin families), which participate in the formation of the intestinal mucosal barrier and play a decisive role in the intestinal barrier [46]. The integrity of tight junctions affects the intestinal inflammatory response [46]. DSS challenge could increase the intestinal permeability, decrease the mRNA and protein expression levels of occludin and claudin-1 in the colon, and destroy the inner barrier of the gut mucosa in mice $[47,48]$. Mice colonised with Lacticaseibacillus rhamnosus have a low serum D-lactic acid level and a low susceptibility to colitis [49]. Similar results were observed in the present study: DSS challenge significantly increased the serum DAO activity and MDA concentrations in the jejunum and colon and decreased the T-AOC, SOD activity, and mRNA expression level of occludin in the colon of the piglets. FMT from Tibetan pigs significantly alleviated the effect of DSS challenge on the serum DAO activity and colonic MDA concentration of the piglets. The outer barrier of the intestinal mucosa consists of normal intestinal microbiota, a mucous layer, and secreted immunoglobulin A. It inhibits the intestinal adhesion and implantation of pathogenic bacteria [50]. Under normal conditions, the microbial count in the loose adhesive mucous layer is higher than that in the strong adhesive mucous layer in the colon of rats. However, the microbiota migrates from the loose adhesive mucous layer to the strong adhesive mucous layer after DSS challenge; this changes the structure and composition of the intestinal microbiota in the loose adhesive mucous layer 
and the strong adhesive mucous layer in the colon [51]. DSSinduced colitis can reduce the Lactobacillus spp. count and increase the $E$. coli count in the intestines. In addition, it can increase the plasma LPS concentration and the expression of proinflammatory factors (such as IL-6, IL-1 $\beta$, IFN$\gamma$, and IL-12) in the colon of rats/mice through Toll-like receptor 4 signals $[52,53]$. A significant reduction in the colonic expression level of MUC2 has been observed in patients with enteritis [54]. MUC2-knockout mice have been found to be more sensitive to DSS-induced colitis and presented with severely damaged mucosa, indicating that MUC2 plays an indispensable role in the gut barrier function $[55,56]$. In the present study, DSS challenge increased the $E$. coli count and propionic acid and total SCFA levels in the colonic digesta, while it decreased the numbers of goblet cells in the colon and the Lactobacillus spp. count and butyric acid levels in the colonic digesta; it also decreased the mRNA expression levels of MUC1 and MUC2 in the jejunum and the mRNA expression levels of MUC2, RegIII $\gamma$, and IL-10 in the colon. It is speculated that the increase in SCFA levels is a compensatory manifestation in the inflammatory model, which is abnormal accumulation and may cause the deepening of inflammation. Moreover, we found that FMT from the Tibetan pigs attenuated the effect of DSS challenge on E. coli count and propionic acid and total SCFA levels in the colonic digesta of piglets. Previous research has revealed that Lactiplantibacillus plantarum administration could enhance the ratio of Firmicutes:Bacteroidetes ratio and diversify the microbial species in the colon of mice [35] and prevent the migration of microorganisms from the loose adhesive mucous layer to the strong adhesive mucous layer in the colon of rats [51]. In addition, probiotic treatment or FMT can reduce the expression and secretion of inflammatory factors (such as IFN- $\gamma$, IL-12, TNF- $\alpha$, IL-6, and IL-1 $\beta$ ) to resist colitis by regulating STAT1, STAT4, or NF- $\kappa$ B signals [57, 58]. As indicated above, it can be speculated that FMT from the Tibetan pigs can attenuate the damage caused by DSS challenge, which can be related to the protective function of the intestinal barrier.

\section{Conclusions}

In summary, DSS infusion can damage the gut health of piglets. However, FMT from Tibetan pigs can attenuate the negative effect of DSS challenge on intestinal development by improving the gut barrier function of the piglets (Figure 2).

\section{Data Availability}

All data generated or analysed during this study are available from the corresponding authors on reasonable request.

\section{Ethical Approval}

All the experimental procedures and animal care were accomplished in accordance with the Guide for the Care and Use of Laboratory Animals provided by the Institutional Animal Care Advisory Committee for Sichuan Agricultural University. All animal protocols conducted in this study were admitted by the Animal Care and Use Committee of Sichuan Agricultural University under permit number DKYB20131704.

\section{Conflicts of Interest}

The authors declare that they have no competing interests.

\section{Authors' Contributions}

DC and XM were responsible for the conceptualisation. HD, $\mathrm{YX}$, and $\mathrm{HY}$ were responsible for the investigation. BY and $\mathrm{JH}$ were responsible for the validation. PZ and JY were responsible for the supervision. All the authors have read and approved the final version of this manuscript. H. Diao, Y. Xiao, and H.L. Yan contributed equally to this work.

\section{Acknowledgments}

The authors sincerely thank Yalan Xu and Fengming Wang for their kind help during the entire study. This study was financially funded by the National Natural Science Foundation of China (31672436) and the National Key Research and Development Program of China (2017YFD0500503).

\section{Supplementary Materials}

Table S1: composition and nutrient level of artificial milk from days 1 to 15 . Table S2: composition and nutrient level of experimental diets from days 16 to 28 . Table S3: composition and nutrient level of experimental diets from days 29 to 56. Table S4: primer sequences and annealing temperature. Table S5: primes and probes for real-time PCR analysis of bacteria. (Supplementary Materials)

\section{References}

[1] L. V. Hooper and J. I. Gordon, "Commensal host-bacterial relationships in the gut,” Science, vol. 292, no. 5519, pp. 1115-1118, 2001.

[2] L. V. Hooper and A. J. Macpherson, "Erratum. Immune adaptations that maintain homeostasis with the intestinal microbiota," Nature Reviews Immunology, vol. 15, no. 5, pp. 329-329, 2015.

[3] J. L. Round and S. K. Mazmanian, "The gut microbiota shapes intestinal immune responses during health and disease," Nature Reviews Immunology, vol. 9, no. 5, pp. 313-323, 2009.

[4] J. Lederberg, "Infectious history," Science, vol. 288, no. 5464, pp. 287-293, 2000.

[5] J. Shen, M. S. Obin, and L. Zhao, "The gut microbiota, obesity and insulin resistance," Molecular Aspects of Medicine, vol. 34, no. 1, pp. 39-58, 2013.

[6] I. Sekirov, S. L. Russell, L. C. Antunes, and B. B. Finlay, "Gut microbiota in health and disease," Physiological Reviews, vol. 90, no. 3, pp. 859-904, 2010.

[7] C. L. Wagner, S. N. Taylor, and D. Johnson, "Host factors in amniotic fluid and breast milk that contribute to gut maturation," Clinical Reviews in Allergy and Immunology, vol. 34, no. 2, pp. 191-204, 2008.

[8] M. Banasaz, E. Norin, R. Holma, and T. Midtvedt, "Increased enterocyte production in gnotobiotic rats mono-associated 
with Lactobacillus rhamnosus GG," Applied and Environmental Microbiology, vol. 68, no. 6, pp. 3031-3034, 2002.

[9] H. A. Gordon and E. Bruckner-Kardoss, "Effect of normal microbial flora on intestinal surface area," The American Journal of Physiology, vol. 201, no. 1, pp. 175-178, 1961.

[10] A. M. O'Hara and F. Shanahan, "The gut flora as a forgotten organ,” EMBO Reports, vol. 7, no. 7, pp. 688-693, 2006.

[11] B. Wostmann and E. Bruckner-Kardoss, "Development of cecal distention in germ-free baby rats," The American Journal of Physiology, vol. 197, no. 6, pp. 1345-1346, 1959.

[12] E. F. Murphy, P. D. Cotter, A. Hogan et al., "Divergent metabolic outcomes arising from targeted manipulation of the gut microbiota in diet-induced obesity," Gut, vol. 62, no. 2, pp. 220-226, 2013.

[13] S. Hapfelmeier, M. A. E. Lawson, E. Slack et al., "Reversible microbial colonization of germ-free mice reveals the dynamics of IgA immune responses," Science, vol. 328, no. 5986, pp. 1705-1709, 2010.

[14] E. Holmes, J. V. Li, J. R. Marchesi, and J. K. Nicholson, "Gut microbiota composition and activity in relation to host metabolic phenotype and disease risk," Cell Metabolism, vol. 16, no. 5, pp. 559-564, 2012.

[15] A. Khoruts and M. J. Sadowsky, "Therapeutic transplantation of the distal gut microbiota," Mucosal Immunology, vol. 4, no. 1, pp. 4-7, 2011.

[16] O. C. Aroniadis and L. J. Brandt, "Fecal microbiota transplantation: past, present and future," Current Opinion in Gastroenterology, vol. 29, no. 1, pp. 79-84, 2013.

[17] H. Diao, H. L. Yan, Y. Xiao et al., "Intestinal microbiota could transfer host gut characteristics from pigs to mice," $B M C$ Microbiology, vol. 16, no. 1, p. 238, 2016.

[18] H. Diao, H. L. Yan, Y. Xiao et al., "Modulation of intestine development by fecal microbiota transplantation in suckling pigs," RSC Advances, vol. 8, no. 16, pp. 8709-8720, 2018.

[19] M. J. Hamilton, A. R. Weingarden, M. J. Sadowsky, and A. Khoruts, "Standardized frozen preparation for transplantation of fecal microbiota for recurrent Clostridium difficile infection," The American Journal of Gastroenterology, vol. 107, no. 5, pp. 761-767, 2012.

[20] B. Zeng, G. Li, J. Yuan, W. Li, H. Tang, and H. Wei, "Effects of age and strain on the microbiota colonization in an infant human flora-associated mouse model," Current microbiol, vol. 67, no. 3, pp. 313-321, 2013.

[21] J. Bassaganya-Riera and R. Hontecillas, "CLA and n-3 PUFA differentially modulate clinical activity and colonic PPARresponsive gene expression in a pig model of experimental IBD," Clinical Nutrition, vol. 25, no. 3, pp. 454-465, 2006.

[22] L. J. Saubermann, P. Beck, Y. P. De Jong et al., "Activation of natural killer T cells by $\alpha$-galactosylceramide in the presence of CD1d provides protection against colitis in mice," Gastroenterology, vol. 119, no. 1, pp. 119-128, 2000.

[23] G. K. Hart and G. J. Dobb, "Effect of a fecal bulking agent on diarrhea during enteral feeding in the critically ill," JPEN Journal of Parenteral and Enteral Nutrition, vol. 12, no. 5, pp. 465468, 2016.

[24] K. S. Kunert, A. S. Tisdale, and I. K. Gipson, "Goblet cell numbers and epithelial proliferation in the conjunctiva of patients with dry eye syndrome treated with cyclosporine," Archives of Ophthalmology, vol. 120, no. 3, pp. 330-337, 2002.

[25] H. Diao, P. Zheng, B. Yu et al., "Effects of benzoic acid and thymol on growth performance and gut characteristics of weaned piglets," Asian-Australasian Journal of Animal Sciences, vol. 28, no. 6, pp. 827-839, 2015.

[26] Y. Zhao, B. Yu, X. Mao et al., "Dietary vitamin D supplementation attenuates immune responses of pigs challenged with rotavirus potentially through the retinoic acid-inducible gene I signalling pathway," British Journal of Nutrition, vol. 112, no. 3, pp. 381-389, 2014.

[27] H. Qi, Z. Xiang, G. Han, B. Yu, Z. Huang, and D. Chen, "Effects of different dietary protein sources on cecal microflora in rats," Afri J Biotechno, vol. 10, pp. 3704-3708, 2011.

[28] N. Fierer, J. A. Jackson, R. Vilgalys, and R. B. Jackson, “Assessment of soil microbial community structure by use of taxonspecific quantitative PCR assays," Applied and Environmental Microbiology, vol. 71, no. 7, pp. 4117-4120, 2005.

[29] H. Diao, P. Zheng, B. Yu et al., "Effects of dietary supplementation with benzoic acid on intestinal morphological structure and microflora in weaned piglets," Livestock Science, vol. 167, pp. 249-256, 2014.

[30] N. Kamada, S. U. Seo, G. Y. Chen, and G. Núñez, "Role of the gut microbiota in immunity and inflammatory disease," Nature Reviews. Immunology, vol. 13, no. 5, pp. 321-335, 2013.

[31] F. Shanahan, "Inflammatory bowel disease: Immunodiagnostics, immunotherapeutics, and ecotherapeutics," Gastroenterology, vol. 120, no. 3, pp. 622-635, 2001.

[32] S. N. S. Murthy, H. S. Cooper, H. Shim, R. S. Shah, S. A. Ibrahim, and D. J. Sedergran, "Treatment of dextran sulfate sodium-induced murine colitis by intracolonic cyclosporin," Digestive Diseases and Sciences, vol. 38, no. 9, pp. 1722-1734, 1993.

[33] X. F. Wang, A. M. Li, J. Li et al., "Low molecular weight heparin relieves experimental colitis in mice by downregulating il$1 \beta$ and inhibiting syndecan-1 shedding in the intestinal mucosa," PLoS One, vol. 8, no. 7, article e66397, 2013.

[34] L. G. Axelsson, E. Landström, and A. C. Bylund-Fellenius, "Experimental colitis induced by dextran sulphate sodium in mice: beneficial effects of sulphasalazine and olsalazine," Alimentary Pharmacology \& Therapeutics, vol. 12, no. 9, pp. 925-934, 1998.

[35] F. Zhang, Y. Li, X. Wang, S. Wang, and D. Bi, “The Impact of Lactobacillus plantarum on the Gut Microbiota of Mice with DSS- Induced Colitis," BioMed Research International, vol. 2019, 10 pages, 2019.

[36] J. M. Chae, W. Heo, H. T. Cho et al., "effects of OrallyAdministered Bifidobacterium animalis subsp. lactis strain BB12 on dextran sodium sulfate-induced colitis in mice," Journal of Microbiology and Biotechnology, vol. 28, no. 11, pp. 1800-1805, 2018.

[37] M. Kverka, Z. Zakostelska, K. Klimesova et al., "Oral administration of Parabacteroides distasonis antigens attenuates experimental murine colitis through modulation of immunity and microbiota composition," Clinical and Experimental Immunology, vol. 163, no. 2, pp. 250-259, 2011.

[38] A. Partty, L. Lehtonen, M. Kalliomaki, S. Salminen, and E. Isolauri, "Probiotic Lactobacillus rhamnosus GG therapy and microbiological programming in infantile colic: a randomized, controlled trial," Pediatric Research, vol. 78, no. 4, pp. 470-475, 2015.

[39] Z. Fesler, E. Mitova, and P. L. Brubaker, "GLP-2, EGF, and the intestinal epithelial IGF-1 receptor interactions in the regulation of crypt cell proliferation," Endocrinology, vol. 161, no. $4,2020$. 
[40] X. Tang, B. Liu, X. Wang, Q. Yu, and R. Fang, "Epidermal growth factor, through alleviating oxidative stress, protect IPEC-J2 cells from lipopolysaccharides-induced apoptosis," International Journal of Molecular Sciences, vol. 19, no. 3, p. 848, 2018.

[41] L. V. Hooper, "Bacterial contributions to mammalian gut development," Trends in Microbiology, vol. 12, no. 3, pp. 129-134, 2004.

[42] T. Sakata and T. Yajima, "Influence of short chain fatty acids on the epithelial cell division of digestive tract," Quarterly Journal of Experimental Physiology, vol. 69, no. 3, pp. 639648, 1984.

[43] X. Guan, B. Stoll, X. Lu et al., "GLP-2-mediated up-regulation of intestinal blood flow and glucose uptake is nitric oxidedependent in TPN-fed piglets 1," Gastroenterology, vol. 125, no. 1, pp. 136-147, 2003.

[44] D. J. Drucker, P. Erlich, S. L. Asa, and P. L. Brubaker, "Induction of intestinal epithelial proliferation by glucagon-like peptide 2," Proceedings of the National Academy of Sciences, vol. 93, no. 15, pp. 7911-7916, 1996.

[45] P. D. Cani, S. Possemiers, T. Van de Wiele et al., "Changes in gut microbiota control inflammation in obese mice through a mechanism involving GLP-2-driven improvement of gut permeability," Gut, vol. 58, no. 8, pp. 1091-1103, 2009.

[46] D. Artis, "Epithelial-cell recognition of commensal bacteria and maintenance of immune homeostasis in the gut," Nature Reviews. Immunology, vol. 8, no. 6, pp. 411-420, 2008.

[47] H. Long, The effects and mechanism of IL-22 on occludin in colonic mucosa of experimental colitis of mice model, Master thesis, Guangxi Medical University, Guangxi, China, 2016.

[48] K. Oh-oka, H. Kono, K. Ishimaru et al., "Expressions of tight junction proteins occludin and claudin-1 are under the circadian control in the mouse large intestine: implications in intestinal permeability and susceptibility to colitis," PLoS One, vol. 9, no. 5, p. e98016, 2014.

[49] F. Yan, L. Liu, H. Cao et al., "Neonatal colonization of mice with LGG promotes intestinal development and decreases susceptibility to colitis in adulthood," Mucosal Immunology, vol. 10, pp. 117-127, 2017.

[50] C. A. Müller, I. B. Autenrieth, and A. Peschel, "Innate defenses of the intestinal epithelial barrier," Cellular and Molecular Life Sciences, vol. 62, no. 12, pp. 1297-1307, 2005.

[51] J. Dicksved, O. Schreiber, B. Willing et al., "Lactobacillus reuteri maintains a functional mucosal barrier during DSS treatment despite mucus layer dysfunction," PLoS One, vol. 7, no. 9, article e46399, 2012.

[52] T. Jilling, D. Simon, J. Lu et al., "The roles of bacteria and TLR4 in rat and murine models of necrotizing enterocolitis," Journal of Immunology, vol. 177, no. 5, pp. 3273-3282, 2006.

[53] Å. Håkansson, N. Tormo-Badia, A. Baridi et al., "Immunological alteration and changes of gut microbiota after dextran sulfate sodium (DSS) administration in mice," Clinical and Experimental Medicine, vol. 15, no. 1, pp. 107-120, 2015.

[54] K. M. A. J. Tytgat, J. W. G. van der Wal, A. W. C. Einerhand, H. A. Büller, and J. Dekker, "Quantitative analysis of MUC2 synthesis in ulcerative colitis," Biochemical and Biophysical Research Communications, vol. 224, no. 2, pp. 397-405, 1996.

[55] M. Van der Sluis, B. A. E. De Koning, A. C. J. M. De Bruijn et al., "Muc2-deficient mice spontaneously develop colitis, indicating that MUC2 is critical for colonic protection," Gastroenterology, vol. 131, no. 1, pp. 117-129, 2006.
[56] J. Petersson, O. Schreiber, G. C. Hansson et al., "Importance and regulation of the colonic mucus barrier in a mouse model of colitis," American Journal of Physiology. Gastrointestinal and Liver Physiology, vol. 300, no. 2, pp. G327-G333, 2011.

[57] F. Liu, The effect and mechanism of levofloxacin and Bifidobacteria on rat visceral sensation in dextran sodium sulfate induced colitis with low grade inflammation, Master thesis, Fujian Medical University, Fujian, China, 2008.

[58] X. Y. Yang, Study on the effects of Lactobacillus in rats with acute ulcerative colitis, Master thesis, Luzhou Medical College, Luzhou, China, 2011. 